\title{
Incidencia de infecciones en el postrasplante renal inmediato
}

\author{
Araceli Faraldo-Cabana, María del Carmen Jiménez-Romero, María Ibáñez-Rebé, María Dolores Rico-del Vas, Ana \\ María Fernández-Cruz, Teresa Lope-Andrea
}

Área de Hospitalización de Nefrología. Hospital Clínico San Carlos de Madrid. España

Como citar este artículo:

Faraldo-Cabana A, Jiménez-Romero MC, Ibáñez-Rebé M, Rico-del Vas MD, Fernández-Cruz AM, Lope-Andrea T. Incidencia de infecciones en el postrasplante renal inmediato. Enferm Nefrol. 2021 0ct-Dic;24(4):379-87

\section{Resumen}

Introducción: El receptor de un trasplante renal es un paciente de alto riesgo para sufrir infección debido a las potenciales complicaciones quirúrgicas y al tratamiento inmunosupresor que reciben.

El desarrollo de infecciones supone un riesgo aumentado de pérdida del injerto y de la mortalidad.

Objetivos: El objetivo principal fue conocer la incidencia de las infecciones más frecuentes en el paciente con un trasplante renal, durante el periodo postrasplante inmediato.

Los objetivos secundarios fueron describir los gérmenes responsables de las infecciones más frecuentes y analizar la relación entre los tipos de infección estudiados y sus posibles factores de riesgo.

Metodología: Estudio observacional retrospectivo en pacientes trasplantados renales entre enero de 2018 y diciembre de 2019 durante el periodo del postrasplante inmediato.

Resultados: La incidencia de infección fue 69,4\%, los síndromes descritos fueron infección del tracto urinario $(48 \%)$, bacteriemia $(9,2 \%)$, infección relacionada con catéter $(8,2 \%)$ e infección por citomegalovirus $(4,1 \%)$. Los microorganimos más frecuentemente implicados, en estos procesos infecciosos fueron Escherichia coli $(16,3 \%)$, Enterococus faecium $(12,2 \%)$ y Enterobacter cloacae $(8,1 \%)$.

\section{Correspondencia:}

Araceli Faraldo Cabana

E-mail:a_faraldo@hotmail.com
Se ha encontrado relación significativa entre la aparición de infección y la duración del ingreso, así como con la presencia o no del antecedente personal de hipertensión arterial. También entre la incidencia de infección relacionada con el catéter venoso central y el tiempo que permaneció insertado.

Conclusiones: La infección más frecuente encontrada en el postrasplante renal inmediato fue la infección del tracto urinario, mientras que el microorganismo más presente habitualmente en los procesos infecciosos fue la Escherichia coli.

PALABRAS CLAVE: trasplante de riñón; infecciones oportunistas; infecciones relacionadas con catéteres; infección orina; bacteriemia.

\section{Incidence of infections in the immediate post- renal transplant period}

\section{Abstract}

Introduction: The kidney transplant recipient is a high-risk patient for infection due to potential surgical complications and immunosuppressive treatment.

The development of infections poses an increased risk of graft loss and mortality.

Objectives: The main objective was to determine the incidence of the most frequent infections in renal transplant patients during the immediate post-transplant period. The secondary objectives were to describe the most frequent infection-producing germs and to analyse the relationship 
between the types of infection studied and their possible risk factors.

Methodology: Retrospective observational study in renal transplant patients between January 2018 and December 2019 during the immediate post-transplant period.

Results: The incidence of infection was $69.4 \%$, the syndromes described were urinary tract infection $(48 \%)$, bacteraemia $(9.2 \%)$, catheter-related infection $(8.2 \%)$ and cytomegalovirus infection $(4.1 \%)$. The main microorganisms involved were Escherichia coli $(16.3 \%)$, Enterococcus faecium $(12.2 \%)$ and Enterobacter cloacae (8.1\%).

A significant relationship was found between the occurrence of infection and the duration of admission, as well as with the presence or absence of a personal history of arterial hypertension. There was also a significant association between the incidence of central venous catheter-related infection and the length of time the catheter was inserted.

Conclusions: The most frequent infection found in the immediate post-renal transplant period was urinary tract infection, while the most common micro-organism present in the infectious processes was Escherichia coli.

KEYWORDS: renal transplantation; opportunistic infections; catheter-related infections; urine infection; bacteraemia.

\section{Introducción}

La Enfermedad Renal Crónica (ERC) evoluciona de forma progresiva hasta sus estadios más avanzados, en los que precisará de algún tratamiento renal sustitutivo. Dada su alta prevalencia es un problema de salud pública a nivel mundial ${ }^{1}$.

El tratamiento de elección para la mayoría de los pacientes es el trasplante renal (TR), ya que, a medio plazo, resulta más eficaz y eficiente que la diálisis por ser la única solución integral2,3. EI TR supone una mejora en la calidad de vida del paciente al reducir la morbimortalidad, con muy buenos resultados en la supervivencia del injerto, que puede llegar hasta el $80 \%$ a los 10 años postrasplante en algunas series ${ }^{4-7}$. A pesar de estos buenos resultados, el receptor de un TR es un paciente de alto riesgo, debido a las potenciales complicaciones quirúrgicas y al impacto de la inmu- nosupresión, que puede aumentar el riesgo de sufrir procesos infecciosos ${ }^{8-11}$.

En España, durante los años 2018 y 2019, periodo que abarca esta investigación, se han realizado 6.736 TR. En las estadísticas se puede observar un aumento del $53,8 \%$ en los últimos 10 años $^{12}$. Durante el periodo de estudio, un $12,5 \%(n=846)$ de los TR se realizaron en la Comunidad de Madrid, de los cuales un 11,5\% ( $n=98)$ se implantaron en el Hospital Clínico San Carlos, donde se ha llevado a cabo este estudio ${ }^{12}$.

Estudios recientes demuestran que padecer una infección puede reducir la supervivencia del injerto a largo plazo ${ }^{13-15}$, especialmente aquellas que se producen durante los tres primeros meses postrasplante ${ }^{10}$. Además, tanto los donantes como los receptores son una población cada vez más envejecida, lo que implica que aumente la mortalidad de origen infeccioso en este contexto ${ }^{4}$.

La localización de la infección más frecuente en los pacientes con un TR es la infección del tracto urinario (ITU) $)^{16,17}$, apareciendo bacteriemia relacionada con $\mathrm{di}$ cha ITU desde un $37,8 \%{ }^{18}$ a un $39 \%{ }^{19} 0$, incluso, hasta en un $55,2 \%{ }^{20}$ de los casos.

La aparición de ITU se puede relacionar con numerosos mecanismos que se ponen en marcha durante la intervención quirúrgica y el periodo postrasplante, como puede ser la interrupción del conducto urinario y el traumatismo del uréter o la presencia de catéteres vesicales; además existen también ciertas anomalías asociadas al trasplante renal, como pueden ser el reflujo vesicouretral y la vejiga neurogénica, que también pueden incrementar el riesgo de ITU ${ }^{16}$. Por último, otro de los factores que predispone para el desarrollo de ITU en el receptor de un TR es la presencia de Diabetes Mellitus ${ }^{10,11,21}$.

Otra de las causas de infección presente en los receptores de un TR es, según la bibliografía ${ }^{22}$, la infección relacionada con catéter. Esto se debe al uso de catéteres venosos centrales (CVC) como práctica habitual, ya que está especialmente indicado en aquellos pacientes que precisan de un acceso venoso por tiempo prolongado, ya sea para la administración de medicación o para la extracción de sangre. En el caso del paciente renal es especialmente necesario ya que su capital venoso suele estar muy dañado, debido a la presencia de fístulas arteriovenosas que limitan el acceso y a las múltiples venopunciones a las que se ven sometidos a lo largo del proceso de su enfermedad. 
La aparición de estas complicaciones asociadas al trasplante, además de provocar los problemas ya descritos, podría incrementar la estancia hospitalaria y los costes asociados a los tratamientos ${ }^{22}$. Por eso, conocer la etiología y la incidencia de la infección puede ayudar a prevenirla, lo que derivaría en una mejora de la atención al paciente, disminuyendo el riesgo de infección, con las consecuencias beneficiosas que ello podría conllevar, tanto para el paciente, como para el sistema sanitario. Asimismo, tras obtener los resultados de este estudio, se podrían identificar posibles medidas de prevención que podrían ayudar a proporcionar al paciente unos cuidados profesionales y de calidad, basados en la evidencia científica.

El objetivo principal del estudio fue determinar la incidencia de las infecciones más frecuentes en el paciente con un TR, durante el periodo postrasplante inmediato.

Los objetivos secundarios fueron, por un lado, identificar los gérmenes responsables de las infecciones más frecuentes, y por otro, analizar la relación entre los tipos de infección estudiados y sus posibles factores de riesgo.

\section{Material y Método}

Se realizó un estudio observacional de cohorte retrospectiva, mediante revisión de las historias clínicas de todos los pacientes, mayores de 18 años, que fueron sometidos a un trasplante de riñón, en el Hospital Clínico San Carlos de Madrid, durante los años 2018 y 2019, estudiándose un total de 98 pacientes.

Fueron registrados los signos de infección que aparecieron durante el periodo inmediato postrasplante, entendiendo éste como el periodo que pasa cada paciente ingresado en la unidad tras ser sometido a un TR.

Se recogió como variable de resultado principal "aparición de infección durante el periodo del postrasplante inmediato", entendiendo infección como la aparición de, al menos una, de las siguientes condiciones:

- Temperatura mayor o igual a $37,9^{\circ} \mathrm{C}$ en una determinación. Durante el periodo de estudio se tomaron 3 determinaciones al día a cada paciente.

- Presencia de bacteriemia, definida ésta, acorde con el Centers for Disease Control and Prevention, como la presencia de bacterias en la sangre, documentadas mediante un cultivo de sangre positivo ${ }^{23}$.

- Presencia de cultivo de punta de CVC positivo.
- Presencia de ITU, entendida ésta como urocultivo positivo.

No se denominó infección nosocomial porque se tuvieron en cuenta la aparición de estos signos de infección desde el mismo día del ingreso, y no 48 horas después, tal y como describe la definición de la infección adquirida en una institución de salud, o infección nosocomial ${ }^{24}$. Como variables de resultado secundarias se recogieron los microorganismos presentes en los hemocultivos, en el urocultivo y en el cultivo de la punta de CVC.

El resto de variables de interés recogidas fueron los datos sociodemográficos de cada paciente, además de sus antecedentes personales y otros posibles factores de riesgo, como el tiempo de estancia hospitalaria, el tiempo de permanencia del CVC y/o de la sonda vesical.

Para el análisis estadístico se realizó un análisis descriptivo. Las variables cualitativas se presentaron como frecuencias absolutas y relativas, y las cuantitativas como media y desviación estándar, en caso de seguir una distribución normal; y en caso contrario, como mediana y rango intercuartílico.

Las variables cualitativas se compararon utilizando el test Ji-cuadrado o prueba exacta de Fisher (en el caso de que más de un $25 \%$ de las frecuencias esperadas fueran menores de 5). Para variables cuantitativas se usó el test de Student o la prueba de Mann-Whitney en caso de que no se distribuyese de forma normal.

Las pruebas se consideraron estadísticamente significativas cuando el $p$-valor fue $<0,05$.

El procesamiento y análisis de datos se realizó con el software IBM SPSS Statistics v21.

El estudio se llevó a cabo de acuerdo con la última versión de la Declaración de Helsinki y las Normas de Buena Práctica Clínica de la Conferencia Internacional de Armonización (GCP/ICH).

Todos los datos personales fueron identificados mediante un código y sólo el investigador puede relacionar dichos datos con el paciente y con su historia clínica. El investigador principal se responsabiliza de custodiar esta información de manera confidencial todo el tiempo que se estipula legalmente, según la legislación española contenida en la Ley Orgánica de Protección de Datos personales y garantía de los derechos digitales (LOPDGDD) 3/2018 de 5 de diciembre. 


\section{Resultados}

Durante el periodo de estudio se realizaron $98 \mathrm{TR}$, un $59,2 \%(n=58)$ fueron hombres, con una edad media de los receptores de $54,9 \pm 13,6$ años, y una edad media de los donantes de $52,2 \pm 16,6$ años. De los 98 pacientes, en un $8,2 \%(n=8)$ no tuvo éxito el trasplante, de ellos, 4 por fallo del injerto en el momento del trasplante, 2 por transplantectomía durante el periodo de estudio, y 2 por fallecimiento del receptor durante el proceso quirúrgico 0 en el periodo del postrasplante inmediato, entendido éste como el periodo que pasa cada paciente ingresado en la unidad tras ser sometido a un TR.

De los trasplantes realizados, un $58,2 \% \quad(n=57)$ procedían de un donante en asistolia, un 38,8\% ( $n=38)$ de un donante en muerte cerebral y un $3,1 \%(n=3)$ de donante vivo.

En los pacientes investigados, presentaron signos de infección un $69,4 \%(n=68)$ de los casos, de los cuales, $26,5 \%(n=18)$ presentaron más de un criterio de infección; entendiendo la misma como presencia de temperatura mayor o igual a $37,9^{\circ} \mathrm{C}$, que se dio en un $30,6 \%(n=30)$ de los pacientes, y/o hemocultivos positivos, presentes en un $9,2 \%(n=9)$ de los individuos, $y / 0$ cultivo positivo al analizar la punta del CVC, que tuvo lugar en un $8,2 \%(n=8)$, o bien, urocultivo positivo, que se encontró en un $48 \%(n=47)$ de los pacientes.

De los receptores que se infectaron, un 54,4\% ( $n=37)$ eran hombres y un $45,6 \%(n=31)$ fueron mujeres, no encontrándose diferencias significativas al compararlos en función de si habían padecido un proceso infeccioso o no $(p=0,14)$.

La edad media de los donantes, en aquellos receptores que se infectaron, fue de $52,3 \pm 16,7$ años, mientras que la edad media del donante, en aquellos receptores que no se infectaron fue $52 \pm 15,8$ años, no encontrándose diferencias significativas en ninguno de los dos casos al compararlos $(p=0,92)$.

De los receptores que se infectaron la edad media fue $55,3 \pm 13,9$ años, mientras que la de los receptores que no se infectaron, la edad media fue $54,2 \pm 13,3$ años, no encontrándose diferencias significativas entre ellos $(p=0,70)$.

Según el tipo de donante, sufrieron un proceso infeccioso durante el periodo de estudio, un 73,7\% $(n=42)$ de los pacientes que recibieron un riñón de donante en asistolia y un $63,1 \%(n=24)$ de los pacientes que recibieron un riñón de donante en muerte cerebral, no existiendo diferencias significativas entre ambos grupos $(p=0,27)$.

Un 30,6\% ( $n=30)$ de los pacientes a estudio padecían Diabetes Mellitus previa al TR, de ellos, el 70\% $(n=21)$ padeció algún proceso infeccioso. El $87,8 \%(n=86)$ del total de los sujetos eran hipertensos, y de ellos presentaron algún criterio de infección un 74,4\% ( $n=64)$, durante el periodo de estudio.

Al relacionar la presencia de diabetes y la infección, no se encontró relación estadísticamente significativa $(p=0,93)$. Sin embargo, sí se encontraron diferencias significativas para padecer una infección, entre los que tenían, o no, hipertensión arterial previa al trasplante $(p=0,007)$.

La duración de la estancia hospitalaria tras el trasplante tuvo una mediana de 13 días (RI: 9-17,2); en el grupo de pacientes que se infectaron la mediana de estancia fue de 15 días (RI: 10,5-20,7), mientras que en los pacientes que no se infectaron, la mediana de estancia fue de 10 días (RI: 8-13,2), encontrándose una diferencia significativa $(p<0,001)$ entre ambos grupos.

En lo que respecta a la sonda vesical y a su tiempo de permanencia, dado el pequeño tamaño muestral de los pacientes que no portaban sonda vesical, la comparación de los dos grupos no puede aportar datos consistentes.

Fueron identificados 24 tipos de microorganismos en los diferentes cultivos.

Los microorganismos con mayor presencia en los episodios de infección de los pacientes fueron: Escherichia coli (16,3\%), Enterococcus faecium (12,2\%), Enterobacter cloacae $(8,1 \%)$, Enterococcus faecalis y Klebsiella pneumoniae $(7,1 \%)$ y Staphylococcus epidermi$\operatorname{dis}(6,1 \%)$.

Algunos de los cultivos presentaron infección polimicrobiana, especialmente en el caso de los urocultivos, ya que de los que resultaron positivos, en un $36,1 \%$ se encontraron dos o más microorganismos.

Las infecciones más habituales fueron las ITU, que aparecieron en un $48 \%(n=47)$ de los individuos. En estas infecciones los microorganismos más frecuentemente implicados fueron las bacterias bacilo gramnegativo $(85,1 \%(n=40))$ siendo el microorganismo más habitual la Escherichia coli $(21,2 \%(n=10))$. 
Los siguientes microorganismos en frecuencia de aparición fueron la Klebsiella pneumoniae que apareció en el $14,8 \%(n=7)$ de los urocultivos positivos y el Enterobacter cloacae, en el $12,7 \%(n=6)$. El resto de microorganismos implicados en la aparición de ITU se puede ver en la tabla 1.

Tabla 1. Etiología de las infecciones del tracto urinario.

\begin{tabular}{|c|c|c|}
\hline $\begin{array}{l}\text { Etiología de las infecciones } \\
\text { del tracto urinario }(n=47)\end{array}$ & n & $\%$ \\
\hline Bacterias & 59 & $125,5 \%$ * \\
\hline Gramnegativas & 40 & $85,1 \%$ \\
\hline Escherichia coli & 10 & $21,2 \%$ \\
\hline Klebsiella pneumoniae & 7 & $14,8 \%$ \\
\hline Enterobacter cloacae & 6 & $12,7 \%$ \\
\hline Pseudomona aeruginosa & 3 & $6,3 \%$ \\
\hline Klebsiella oxytoca & 3 & $6,3 \%$ \\
\hline Citrobacter freundii & 2 & $4,2 \%$ \\
\hline Serratia marcescens & 2 & $4,2 \%$ \\
\hline Enterobacter aerogenes & 2 & $4,2 \%$ \\
\hline Morganella morganii & 1 & $2,1 \%$ \\
\hline Proteus penneri & 1 & $2,1 \%$ \\
\hline Proteus vulgaris & 1 & $2,1 \%$ \\
\hline Citrobacter amalonaticus & 1 & $2,1 \%$ \\
\hline Enterobacter kobei & 1 & $2,1 \%$ \\
\hline Grampositivas & 19 & $40,4 \%$ \\
\hline Enterococcus faecium & 10 & $21,2 \%$ \\
\hline Enterococcus faecalis & 7 & $14,8 \%$ \\
\hline Staphylococcus epidermidis & 2 & $4,2 \%$ \\
\hline Hongos & 8 & $17 \%$ \\
\hline Candida albicans & 5 & $10,6 \%$ \\
\hline Candida glabrata & 2 & $4,2 \%$ \\
\hline Candida krusei & 1 & $2,1 \%$ \\
\hline Virus & 0 & $0 \%$ \\
\hline Episodios de etiología polimicrobiana & 17 & $36,1 \%$ \\
\hline
\end{tabular}

* Los porcentajes superiores al $100 \%$ son debidos al gran número de infecciones polimicrobianas encontradas en esta investigación.

La incidencia de Citomegalovirus (CMV) se estudió mediante comprobación de la carga viral en sangre tras la realización del TR. Se tomaron muestras analíticas al $97 \%(n=95)$ de los sujetos, siendo positivos el $4,2 \%$ $(n=4)$.
En la población estudiada, el 97,9\% ( $n=96)$ eran portadores de una sonda vesical que permaneció insertada una mediana de 7 días (RI: 7-7). En los pacientes que presentaron ITU, la sonda vesical estuvo insertada una mediana de 7 días (RI: 6,7-7), y en los que no presentaron ITU, el tiempo de inserción de la sonda vesical también tuvo una mediana de 7 días (RI: 7-7), la diferencia entre ambos grupos no es estadísticamente significativa $(p=0,56)$.

En lo que se refiere a los datos encontrados sobre el CVC, un $73,4 \% \quad(n=72)$ de la población estudiada portaba un CVC. En los pacientes que no presentaron infección de la punta $(88,9 \%(n=64))$, el CVC permaneció insertado una mediana de 6 días (RI: 4-8); mientras que en los que sí presentaron infección de la punta $(11,1 \%(n=8))$, la mediana del tiempo de inserción fue de 9,5 (RI: 7,2-18,5), resultando una diferencia estadísticamente significativa $(p=0,03)$ al compararlos.

En las puntas de CVC que presentaron infección $(n=8)$, el microorganismo más frecuente fue, en primer lugar, el Staphylococcus epidermidis, que se encontró en 4 de las puntas de catéter, seguido por la Escherichia coli y Staphylococcus haemolyticus, ambas con una frecuencia de 2.

La aparición de bacteriemia, definida como hemocultivos positivos, tuvo una incidencia del $9,2 \%(n=9)$. El microorganismo más frecuentemente implicado fue la Escherichia coli que apareció en un $44,4 \%(n=4)$ de los hemocultivos positivos.

El resto de microorganismos encontrados, tanto en el análisis de la punta de catéter como en los hemocultivos positivos, se pueden ver en la tabla 2.

\section{Discusión}

Según demuestra la bibliografía, las infecciones influyen negativamente en la supervivencia del injerto tras un trasplante renal, llegando a convertirse en una de las principales causas de mortalidad, especialmente durante el primer año postrasplante ${ }^{25-27}$. Para poder conseguir un equilibrio adecuado entre la inmunosupresión y el riesgo de infección, es muy importante que el tratamiento sea ajustado individualmente y monitorizado regularmente, ya que los pacientes trasplantados son especialmente vulnerables a padecer infecciones ${ }^{27}$.

Lograr una adecuada inmunosupresión tiene un precio, por lo que corregir otros factores modificables, como pue- 
Tabla 2. Etiología de las bacteriemias (identificadas por HC positivos) y del cultivo de la punta del CVC.

\begin{tabular}{|c|c|c|c|c|c|}
\hline $\begin{array}{l}\text { Etiología de las bacteriemias } \\
(n=9)\end{array}$ & $\mathbf{n}$ & $\%$ & $\begin{array}{l}\text { Etiología de la contaminación de punta } \\
\text { de catéter }(n=8)\end{array}$ & n & $\%$ \\
\hline Bacterias & 10 & $111,1 \% *$ & Bacterias & 13 & $162,5 \%$ * \\
\hline Gramnegativas & 8 & $88,8 \%$ & Gramnegativas & 3 & $37,5 \%$ \\
\hline Escherichia coli & 4 & $44,4 \%$ & Escherichia coli & 2 & $25 \%$ \\
\hline Enterobacter cloacae & 1 & $11,1 \%$ & Enterobacter cloacae & 1 & $12,5 \%$ \\
\hline Pseudomona aeruginosa & 1 & $11,1 \%$ & & & \\
\hline Klebsiella oxytoca & 1 & $11,1 \%$ & & & \\
\hline Citrobacter freundii & 1 & $11,1 \%$ & & & \\
\hline Grampositivas & 2 & $22,2 \%$ & Grampositivas & 10 & $125 \%$ * \\
\hline Enterococcus faecium & 1 & $11,1 \%$ & Staphylococcus epidermidis & 4 & $50 \%$ \\
\hline \multirow{5}{*}{$\begin{array}{l}\text { Staphylococcus coagulasa } \\
\text { negativo }\end{array}$} & \multirow[t]{5}{*}{1} & \multirow[t]{5}{*}{$11,1 \%$} & Staphylococcus Haemolyticus & 2 & $25 \%$ \\
\hline & & & Enterococcus faecium & 1 & $12,5 \%$ \\
\hline & & & Staphylococcus aureus & 1 & $12,5 \%$ \\
\hline & & & Corynebacterium & 1 & $12,5 \%$ \\
\hline & & & Streptococcus agalactiae & 1 & $12,5 \%$ \\
\hline Hongos & 0 & & Hongos & 0 & \\
\hline Virus & 0 & & Virus & 0 & \\
\hline $\begin{array}{l}\text { Episodios de etiología } \\
\text { polimicrobiana }\end{array}$ & 1 & $11,1 \%$ & Episodios de etiología polimicrobiana & 3 & $37,5 \%$ \\
\hline
\end{tabular}

* Los porcentajes superiores al 100\% son debidos al gran número de infecciones polimicrobianas encontradas en esta investigación.

de ser, por ejemplo, el tiempo que permanece insertado el CVC, o el cuidado con el que se manejen los catéteres tanto venosos como urinarios, puede resultar muy útil a la hora de prevenir la infección 8.28 .

Debido al escaso tamaño muestral de los pacientes que recibieron un riñón de donante en vivo, no es comparable con los otros dos grupos.

Mientras que la bibliografía previa ${ }^{10}$ destaca la relación entre la presencia de diabetes y la infección, no es el caso del presente estudio, donde no se encontró esta relación.

En lo que respecta a las diferencias estadísticamente significativas que se han encontrado al relacionar la HTA con la infección, hay que ser cautelosos a la hora de tener en cuenta este hecho, ya que el porcentaje de población sin HTA es muy bajo en nuestra muestra, por lo que los resultados podrían no ser comparables.

Los antecedentes bibliográficos ${ }^{11}$ son acordes con la diferencia estadísticamente significativa que se encontró al relacionar la duración de la estancia hospitalaria tras el trasplante con la aparición de infección, este hecho podría deberse a que los pacientes que presentan complicaciones postoperatorias (entre las que se incluyen las infecciones, entre otras), pueden ver aumentado su tiempo de estancia hospitalaria para la resolución de dichos problemas; sería un aspecto interesante para investigar en futuros proyectos.

Siguiendo la línea de estudios anteriores ${ }^{16,22}$, las infecciones más habituales fueron las ITU, siendo el microorganismo más frecuente la Escherichia coli, dato que coincide con la bibliografía existente ${ }^{10,11,17,29,30}$, en la que también se describe como la causa más frecuente de ITU en la población general ${ }^{29}$.

La incidencia de CMV encontrada resulta muy baja en comparación con investigaciones previas, cuyos resultados de infección por CMV son mucho más elevados, llegando desde el $30 \%{ }^{30}$ al $34,7 \%{ }^{31}$ según estudios consultados. No se dispone de información sobre si el paciente tenía muestra de CMV positiva previa al trasplante. 
Un aspecto a resaltar es el alto porcentaje de infecciones polimicrobianas que se encontraron en este estudio, sería interesante en estudios posteriores tratar de descartar una posible contaminación de la muestra.

La relación significativa encontrada entre el tiempo que permanece insertado el CVC y la infección es acorde con la bibliografía existente, ya que estudios previos ${ }^{11,22}$ habían demostrado con anterioridad que cuanto más tiempo pasa el CVC insertado mayor es el riesgo de contaminación.

Las complicaciones infecciosas del CVC, según la bibliografía ${ }^{32,33}$, están muy relacionadas con el trabajo de enfermería ya que pueden ser minimizadas mediante un protocolo apropiado que englobe la forma correcta de manipulación, la realización de las curas, la heparinización, la administración de medicamentos y fluidos y las extracciones sanguíneas.

A pesar del esfuerzo de los autores por intentar paliarlas, el estudio presenta algunas limitaciones. En primer lugar, puede haber pacientes que hayan tenido una infección transitoria (que diesen falso negativo en los cultivos o que cursarse de forma asintomática) y que no hayan quedado registrados, lo que podría resultar en una infraestimación de la verdadera incidencia de la infección. En segundo lugar, los datos perdidos han sido mínimos ya que se han consultado todas las bases de datos disponibles, pero algunas historias clínicas no tenían registrada la información necesaria para este estudio. En tercer lugar, el periodo de seguimiento de cada paciente ha abarcado sólo el tiempo que ha permanecido hospitalizado tras la realización del TR, sin incluir el tiempo del postrasplante en el domicilio, por lo que nos planteamos realizar un estudio posterior, en el que el periodo de estudio sea más amplio. Además, en este mismo sentido hay que tener en cuenta que el periodo de seguimiento de cada paciente ha sido variable, ya que dependió del momento del alta médica, por lo que también en futuras investigaciones sería interesante establecer un periodo determinado para que el seguimiento sea el mismo para cada paciente. Y, por último, este estudio se ha realizado en una cohorte de un hospital terciario (unicéntrico), por lo que se debe tener precaución a la hora de generalizar los datos.

Como conclusiones se desprende que, tras el análisis de los datos, la infección más frecuente encontrada en esta investigación fue la ITU, mientras que el microorganismo más presente habitualmente en los procesos infecciosos fue la Escherichia coli.
Destacar que a partir de los resultados obtenidos en esta investigación se pueden establecer medidas que ayuden a disminuir el riesgo de infección en los pacientes con un TR.

Recepción: 05-05-21

Aceptación: 09-11-21

Publicación: 30-12-21

\section{Bibliografía}

1. Kidney Disease: Improving Global Outcomes (KDIGO) CKD Work Group. KDIG0 2012 clinical practice guideline for the evaluation and management of chronic kidney disease. Kidney Int Suppl. Jun 2013;3(1):1-150.

2. Faraldo Cabana $A$, Ibáñez Rebé $M$, Del Río Rodríguez M, Esteban Polonio C, Lope Andrea T, Muñoz Jiménez D. Frecuencia de los diagnósticos de enfermería en el primer ingreso del paciente con un trasplante renal reciente. Enferm Nefrol. 2017;20(1):76-81.

3. Berlango Jiménez, J. Indicaciones del trasplante renal. Preparación preoperatoria. En: Force, A. La Enfermería y el trasplante de órganos. Madrid. Ed. Panamericana. 2004. p. 89.

4. Hernández Marrero D, González-Molina Alcaide M. Resultados Globales del Trasplante Renal. En: Lorenzo V, López Gómez JM. Nefrología al Día [Internet]. [consultado 30 Jun 2020]. Disponible en: http:// www.revistanefrologia.com/es-monografias-nefrologia-dia-articulo-resultados-globales-del-trasplante-renal-58.

5. 0jo A0, Morales JM, González-Molina M, Steffick $D E$, Luan FL, Merion RM, et al. Comparison of the long-term outcomes of kidney transplantation: USA versus Spain. Nephrol Dial Transplant. 2013;28(1):213-20.

6. Bell R, Farid S, Pandanaboyana S, Upasani V, Baker $R$, Ahmad N. The evolution of donation after circula- 
tory death renal transplantation: a decade of experience. Nephrol Dial Transplant. 2019;34(10):178898.

7. Lodhi SA, Lamb KE, Meier-Kriesche HU. Solid organ allograft survival improvement in the United States: the long-term does not mirror the dramatic short-term success. Am J Transplant. 2011 Jun;11(6):1226-35.

8. Chen HS, Wang FD, Lin Y-C, Huang LJ, Liu CY. Risk factors for central venous catheter-related infections in general surgery. J Microbiol Immunol Infection. 2006;39:231-6.

9. Hammarskjöld F, Wallén G, Malmvall BE. Central venous catheter infections at a county hospital in Sweden: a prospective analysis of colonization, incidence of infection and risk factors. Acta Anaesthesiol Scand. 2006;50:451-60.

10. Brar S, Wang Y, Cannitelli A, Lambadaris M, Li Y, Famure 0 , et al. Bacteremia in kidney transplant recipients: Burden, causes, and consequences. Clinical Transplantation. 2019; 33:e13479.

11. Galindo Sacristán P, Pérez Marfil A, Osorio Moratall JM, de Gracia Guindo C, Ruiz Fuentes C, CastiIla Barbosa YA, et al. Predictive factors of Infection in the 1st year after kidney transplantation. Transpl Proc. 2013; 45:3620-3.

12. Actividad de donación y trasplante renal. España 2019. Ministerio de Sanidad, Servicios Sociales e Igualdad. [Consultado 04 Jul 2020]. Disponible en: http://www.ont.es/infesp/Memorias/Actividad_de_ Donaci\%C3\%B3n_y_Trasplante_Renal_2019.pdf.

13. Al-Hasan MN, Razonable RR, Kremers WK, Baddour LM. Impact of Gram-negative bloodstream infection on long-term allograft survival after kidney transplantation. Transplantation. 2011;91(11):1206-10.

14. Parasuraman R, Abouljoud M, Jacobsen G, Reddy $G$, Koffron A, Venkat KK. Increasing trend in infection-related death-censored graft failure in renal transplantation. Transplantation. 2011;91(1):94-9.

15. Skov Dalgaard L, Nørgaard M, Povlsen JV, Morrissey 0 , Jespersen $B$, Jensen-Fangel $S$, et al. Risk and prognosis of bacteremia and fungemia among first-time kidney transplant recipients: a popula- tion-based cohort study. Infect Dis (Lond). 2017 Apr;49(4):286-95.

16. Rodríguez Martínez R, Bacallao Méndez R, Gutiérrez García F, Fonseca Hernández D. Complicaciones del trasplante renal en el Instituto de $\mathrm{Ne}$ frología. 2001-2005. Rev Soc Esp Enferm Nefrol. 2010;13(1):7-15.

17. Kritikos $A$, Oriol M. Bloodstream infections after solid-organ transplantation. Virulence. 2016;7(3):32940.

18. Silva M, Marra AR, Pereira CA, Medina-Pastana J0, Camargo LF. Bloodstream infection after kidney transplantation: epidemiology, microbiology, associated risk factors, and outcome. Transplantation. 2010;90(5):581-7.

19. Moreno A, Cervera C, Gavalda J, Rovira M, de la Camara R, Jarque I, et al. Bloodstream infections among trasplant recipients: results of a nationwide surveillance in Spain. Am J Trasplant. 2007;7(11):2579-86.

20. Al-Hasan MN, Razonable RR, Eckel-Passow JE, Baddour LM. Incidence rate and outcome of gram-negative bloodstream infection in solid organ transplant recipients. Am J Trasplant. 2009;9(4):835-43.

21. Tolkoff-Rubin NE, Rubin RH. The infectious disease problems of the diabetic renal transplant recipient. Infect Dis Clin North Am. 1995;9(1):117-30.

22. Moghadamyeghaneh $Z$, Chen LJ, Alameddine M, Gupta AK, Burke GW, Ciancio G. Never events and hospital-acquired conditions after kidney transplant. Can Urol Assoc J. 2017 Novi11(11):E431-E436.

23. Garner JS, Jarvis WR, Emori TG, Horan TC, Hughes JM. CDC definitions for nosocomial infections, 1988. Am J Infect Control. 1988;16(3):128-40.

24. Pujol M, Limón E. Epidemiología general de las infecciones nosocomiales. Sistemas y programas de vigilancia. Enferm Infecc Microbiol Clin. 2013;31(2):108-13.

25. US Renal Data System. USRDS. 2011 annual data report: atlas of chronic kidney disease and end-stage renal disease in the United States. Bethesda, 
Maryland: National Institutes of Health, National Institute of Diabetes and Digestive and Kidney Diseases; 2011.

26. Hernández D, Moreso F. Has patient survival following renal transplantation improved in the era of modern immunosuppression? Nefrología. 2013;33:171-80.

27. Martorell J. Mecanismos de respuesta inmune e infección. En: Aguado García J, Fortún Abete J, Gavaldá Santapau J, Pahissa Berga A, de la Torre Cisneros J. Infecciones en pacientes trasplantados. $3 \mathrm{rd}$ ed. 2009;49-59.

28. Figueroa-Sánchez GE, Arreola JM, Morales-Buenrostro $L E$. Factores de riesgo para infección de vías urinarias en el periodo postrasplante renal temprano. Revista Mexicana de Trasplantes. 2012;1(1):228.

29. Cong-Tat C, Ming-Ji L, Chia-Wen L, Nan-Yao L, Shen-Shin C, Ching-Chi L, et al. Community-onset bacteremia in kidney transplant recipients: The recipients fare well in terms of mortality and kidney injury. Journal of Microbiology, Immunology and Infection. 2016;49:685-91.
30. García-Prado ME, Cordero $E$, Cabello V, Pereira $P$, Torrubia FJ, Ruíz M, et al. Complicaciones infecciosas en 149 receptores de trasplante renal consecutivos. Enferm Infecc Microbiol Clín. 2009;27(1):22-7.

31. López-Oliva M0, Flores J, Madero R, Escuin F, Santana MJ, Bellón T, et al. Cytomegalovirus infection after kidney transplantation and long-term graft loss. Nefrologia. 2017 Sep-0ct; 37(5):515-25.

32. 0' Grady NP, Alexander M, Dellinger EP, Gerberding JL, Heard S0, Maki DG, et al. Guidelines for the Prevention of Intravascular Catheter-Related Infections. Centers for Disease Control and Prevention. MMWR 2002;51(No.RR-10):13-6.

33. Elliot T, Timsit J-F. EPIC3: Guidelines for preventing infections associated with the use of intravascular access devices. J Hosp Infect. 2014 Jul;87(3):182.

Este artículo se distribuye bajo una Licencia Creative Commons Atribución-NoComercial 4.0 Internacional. https://creativecommons.org/licenses/by-nc/4.0/ 